\title{
Cancer-associated fibroblasts enhance the migration ability of ovarian cancer cells by increasing EZH2 expression
}

\author{
LINJUAN XU ${ }^{1 *}$, QINGCHUN DENG $^{1,2^{*}}$, YINGLIAN PAN $^{3}$, MINGGANG PENG $^{1}$,

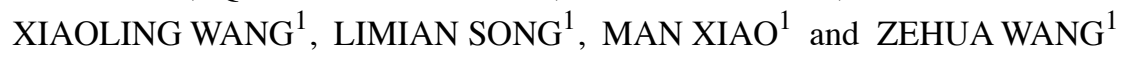 \\ ${ }^{1}$ Department of Obstetrics and Gynecology, Union Hospital, Tongji Medical College, \\ Huazhong University of Science and Technology, Wuhan, Hubei 430022; Departments of ${ }^{2}$ Obstetrics and Gynecology \\ and ${ }^{3}$ Rehabilitation, Southwest Hospital, Third Military Medical University, Chongqing 400038, P.R. China
}

Received July 25, 2013; Accepted October 15, 2013

DOI: 10.3892/ijmm.2013.1549

\begin{abstract}
The tumor microenvironment is thought to affect malignant transformation and tumor progression. The histone methyltransferase, enhancer of zeste homologue 2 (EZH2), has recently been suggested to play a critical role in the tumorigenesis of several types of human cancer. The aim of this study was to investigate the effects of cancer-associated fibroblasts (CAFs) on the expression of EZH2 and the migration ability of ovarian cancer cells, in order to explore the link between the tumor microenvironment and epigenetic regulation. The ovarian cancer cell lines, A2780, SKOV3 and ES2, were indirectly co-cultured with primary ovarian CAFs or normal fibroblasts (NFs). The migration ability of the ovarian cancer cells was determined by Transwell migration assay. The expression levels of EZH2 were assessed by quantitative reverse transcription PCR (qRT-PCR) and western blot analysis. The A2780-shEZH2 cells (A2780 cells transfected with shRNA targeting EZH2) were indirectly co-cultured with CAFs or NFs, and the changes in the expression levels of EZH2 and the migration ability of the cells were detected. The migration ability of the A2780, SKOV3 and ES2 cells co-cultured with CAFs was significantly enhanced $(\mathrm{P}<0.05)$ compared with the NF group and the cells cultured alone. The expression of EZH2 in the A2780, SKOV3 and ES2 cells was significantly increased following co-culture with CAFs $(\mathrm{P}<0.001)$ compared with the cells cultured alone but not
\end{abstract}

Correspondence to: Professor Zehua Wang, Department of Obstetrics and Gynecology, Union Hospital, Tongji Medical College, Huazhong University of Science and Technology, 1277 Jiefang Avenue, Wuhan, Hubei 430022, P.R. China

E-mail: zehuawang@163.net

${ }^{*}$ Contributed equally

Key words: ovarian cancer, cancer-associated fibroblasts, enhancer of zeste homologue 2, migration those cultured with NFs. The migration ability of the A2780shEZH 2 cells was not significantly increased following co-culture with CAFs $(\mathrm{P}>0.05)$. Our data indicate that CAFs enhance the migration ability of ovarian cancer cells partly by increasing EZH2 expression.

\section{Introduction}

Ovarian cancer is the most lethal gynecological malignancy worldwide. The high mortality rate of patients with ovarian cancer is attributed to the late diagnosis of this type of tumor. Approximately $70 \%$ of patients with ovarian cancer are first diagnosed in the advanced stage of disease $(1,2)$. The 5 -year survival rate of patients with advanced-stage ovarian cancer is only $30 \%$. In women, $>80 \%$ of malignant ovarian tumors are of epithelial origin and the prognosis of epithelial ovarian carcinoma (EOC) is poor (3).

The tumor microenvironment is composed of blood and lymphatic vessels, tissue fluid, fibroblasts, inflammatory cells and a large number of extracellular matrices, which are essential for tumor cell growth $(4,5)$. Numerous studies have found that cancer-associated fibroblasts (CAFs) play an important role in a wide variety of tumors, such as breast, prostate, esophageal, pancreatic and lung cancer by promoting the initiation, proliferation, invasion and metastasis of cancer cells; however, the underlying mechanisms are not yet fully understood (6-9). In our previous studies, we found that the abundance of CAFs in ovarian cancer is associated with the advanced stage of the tumor, lymph node metastasis and omental metastasis. We successfully isolated primary ovarian CAFs and normal fibroblasts (NFs) $(10,11)$.

The enhancer of zeste homologue 2 (EZH2) is a subunit of polycomb repressive complex 2 (PRC2), which catalyzes the methylation of histone 3 lysine 27 (H3K27). EZH2 has been shown to be overexpressed in many types of cancer, which contributes to the silencing of tumor suppressor genes and is involved in the initiation and progression of tumors (12-14). The aim of this study was to investigate the effects of CAFs on the migration ability of ovarian cancer cells and the involvement of EZH2 in this process, as well as the underlying mechanisms involved. 


\section{Materials and methods}

Cell culture. Three ovarian cancer cell lines (A2780, SKOV3 and ES2) were used in the present study. A2780 cells were obtained from the China Center for Type Culture Collection (CCTCC; Wuhan, China) and SKOV3 and ES2 cells were purchased from the Cell bank of China Center for Type Culture Collection, Chinese Academy of Sciences (CTCCCAS, Shanghai, China). The A2780 cells stably transfected with shRNA targeting EZH2 (shEZH2; A2780-shEZH2 cells) or shNC (negative control; A2780-shNC cells) were previously established in our laboratory (15). Primary CAFs in EOC and ovarian NFs were isolated and identified as described in our previous study (10). The A2780, ES2, A2780-shEZH2 and A2780-shNC cells were cultured in RPMI-1640 medium (Invitrogen, Carlsbad, CA, USA) containing 10\% fetal bovine serum (FBS; Gibco, Carlsbad, CA, USA). The SKOV3 cells were cultured in Dulbecco's modified Eagle's medium (DMEM; Invitrogen) supplemented with 10\% FBS. Primary CAFs and NFs were cultured in DMEM/F12 supplemented with $20 \%$ FBS. All the cell lines were cultured at $37^{\circ} \mathrm{C}$, in a humidified atmosphere of $5 \% \mathrm{CO}_{2}$.

Indirect co-culture. The A2780, SKOV3 and ES2 cells were indirectly co-cultured with primary ovarian CAFs or NFs in 6-well Transwell chamber plate with pores of $0.4 \mu \mathrm{m}$ in diameter. Fibroblasts were seeded in the lower compartment $\left(4 \times 10^{4}\right.$ cells/well) and EOC cells in the upper compartment $\left(4 \times 10^{4}\right.$ cells/well). These cells were indirectly co-cultured for 7 days. The EOC cells cultured alone represented the blank group which was used as the control. The experiment was repeated 3 times separately.

Quantitative real-time polymerase chain reaction ( $q R T-P C R$ ). Total RNA from the cells was extracted using TRIzol Reagent (Invitrogen) and reverse transcribed into complementary DNA (cDNA) using the Reverse Transcription kit (Toyobo, Osaka, Japan). The sequences of the primers used were as follows: EZH2, 5'-CTCCCGCTGAGGATGTGGATAC-3' (forward) and 5'-GGCTCCACAAGTAAGACAGAGGTC-3' (reverse); $\beta$-actin, 5'-GCCAACACAGTGCTGTCTGG-3' (forward) and 5'-GCTCAGGAGGAGCAATGATCTTG-3' (reverse). PCR was performed according to the manufacturer's instructions using SYBR-Green PCR Master Mix (Toyobo). The amplification protocols were as follows: $95^{\circ} \mathrm{C}$ for $60 \mathrm{sec}$; 40 cycles of $95^{\circ} \mathrm{C}$ for $15 \mathrm{sec}, 57^{\circ} \mathrm{C}$ for $15 \mathrm{sec}$ and $72^{\circ} \mathrm{C}$ for $45 \mathrm{sec}$. EZH2 mRNA was normalized to $\beta$-actin amplification. Relative quantification was performed using the comparative threshold cycle $(\mathrm{Ct})$ method $\left(2^{-\Delta \Delta C t}\right)$ as previously described (16). All reactions were performed in triplicate.

Western blot analysis. Total protein from the cells was extracted using radioimmune precipitation assay (RIPA) buffer. The protein concentration was measured by the bicinchoninic acid (BCA) assay. Fifty micrograms of total protein were separated by $10 \%$ sodium dodecyl sulfate-polyacrylamide gel electrophoresis (SDS-PAGE) and transferred onto a nitrocellulose membrane. The membranes were incubated at $4^{\circ} \mathrm{C}$ overnight with the following primary antibodies: mouse anti-EZH2 polyclonal antibody (1:500 dilution; Cell Signaling Technology,
Beverly, MA, USA), or mouse anti- $\beta$-actin polyclonal antibody (1:500 dilution; Santa Cruz Biotechnology, Inc., CA, USA). The primary antibodies were detected by incubating with horseradish peroxidase-conjugated anti-mouse secondary antibody (1:5,000 dilutions; Santa Cruz Biotechnology, Inc.) for $2 \mathrm{~h}$ at room temperature and visualized using an ECL system (Beyotime, Shanghai, China) by exposure to X-ray film. The protein bands were quantified using Quantity One software (Bio-Rad, Hercules, CA, USA). The experiment was repeated 3 times separately.

Cell migration assay. The 24-well Transwell chamber plate with pores of $8 \mu \mathrm{m}$ in diameter was used to determine the migration ability of the cells. Cells $\left(5 \times 10^{4}\right.$ cells/well $)$ were resuspended in $100 \mu \mathrm{l}$ culture medium, and then added to the Transwell inserts (Corning Glass Works; Corning, NY, USA). The lower chamber beneath the insert membrane was supplemented with $600 \mu \mathrm{l}$ corresponding medium following incubation for $16 \mathrm{~h}$, and the migrated cells on the lower surface of the membrane were fixed with $95 \%$ ethanol and stained with crystal violet followed by cell counting. All assays were performed in triplicate.

Statistical analysis. Statistical analysis was performed using SPSS 13.0 statistical software (SPSS, Chicago, IL, USA). All numerical data are expressed as the means \pm standard deviation. Statistical significance of the differences was analyzed using a two-tailed Student's t-test or one-way ANOVA. A value of $\mathrm{P}<0.05$ was considered to indicate a statistically significant difference.

\section{Results}

CAFs enhance the migration ability of ovarian cancer cells. Ovarian cancer cells were cultured alone or indirectly co-cultured with CAFs or NFs and their migration ability was assessed. Compared with the cells cultured alone, the number of A2780, SKOV3 and ES2 cells co-cultured with CAFs penetrating the membrane increased by $1.97-, 3.46-$ and 1.49-fold, respectively; compared with the cells cultured alone. The number of A2780, SKOV3 and ES2 cells co-cultured with NFs penetrating the membrane increased by 1.19-, 1.54and 1.13-fold, respectively (Fig. 1). The migration ability of the cells co-cultured with CAFs was markedly enhanced in comparison to the blank group and the difference was statistically significant $\left(\mathrm{P}_{\mathrm{A} 2780}<0.001, \mathrm{P}_{\mathrm{SKOV}_{3}}<0.001\right.$ and $\left.\mathrm{P}_{\mathrm{ES} 2}<0.05\right)$; the migration ability of the cells in the NF group (apart from ES2 cells) was markedly enhanced in comparison to the blank group $(\mathrm{P}<0.001)$.

CAFs increase the expression of EZH2 in ovarian cancer cells. The alterations in the expression levels of EZH2 in the co-cultured ovarian cancer cell lines were assessed by qRT-PCR and western blot analysis. The results of qRT-PCR revealed that the CAFs significantly increased the mRNA expression of EZH2 in the ovarian cancer cells. Compared with the cells cultured alone, the mRNA expression of EZH2 in the A2780, SKOV 3 and ES2 cells co-cultured with CAFs increased by 3.41-, 4.12-, 4.85-fold, respectively $\left(\mathrm{P}_{\mathrm{A} 2780}<0.001, \mathrm{P}_{\mathrm{SKOV} 3}<0.001\right.$ and $\mathrm{P}_{\mathrm{ES} 2}<0.001$ ); the ovarian NFs did not significantly increase 
A

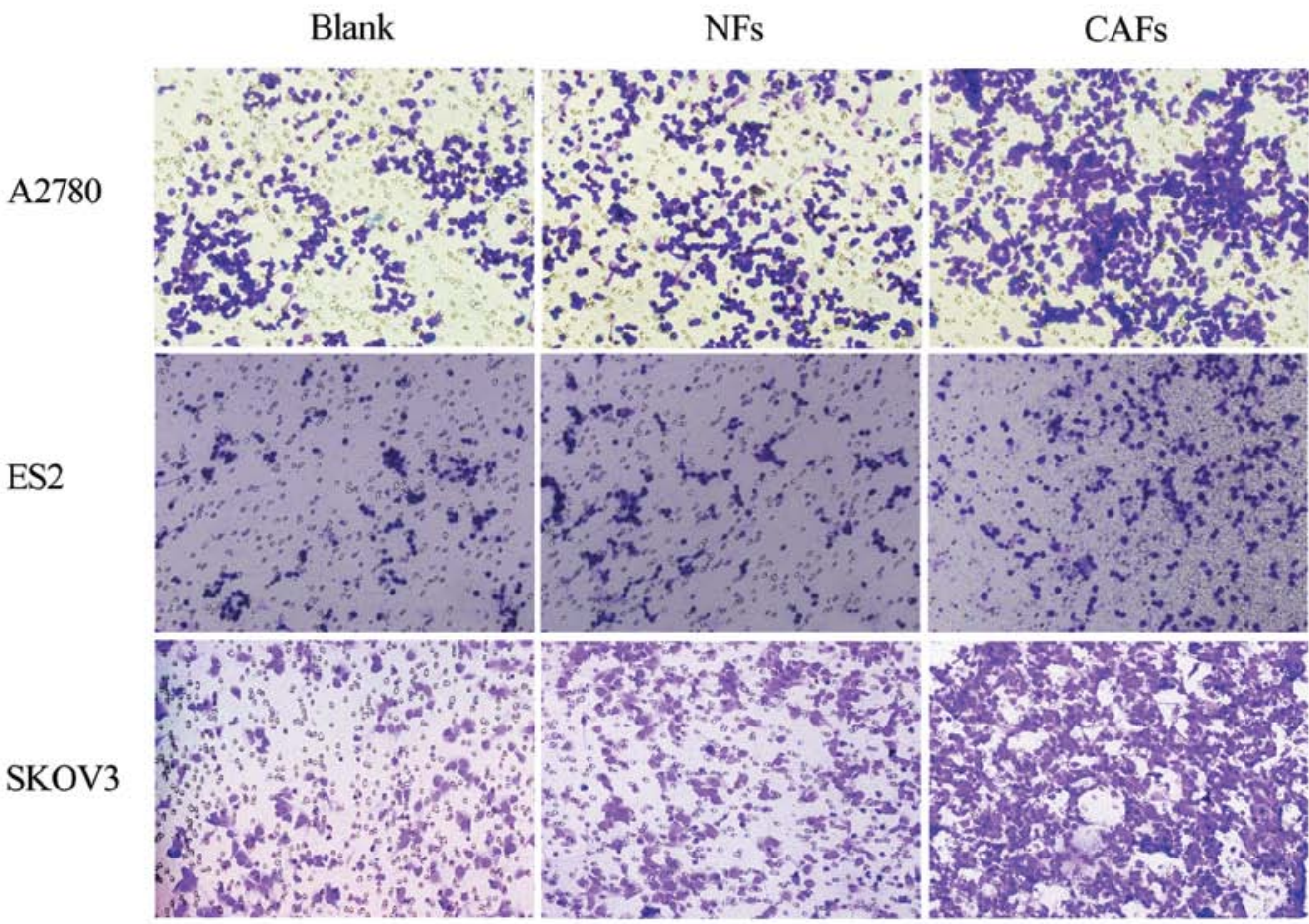

B

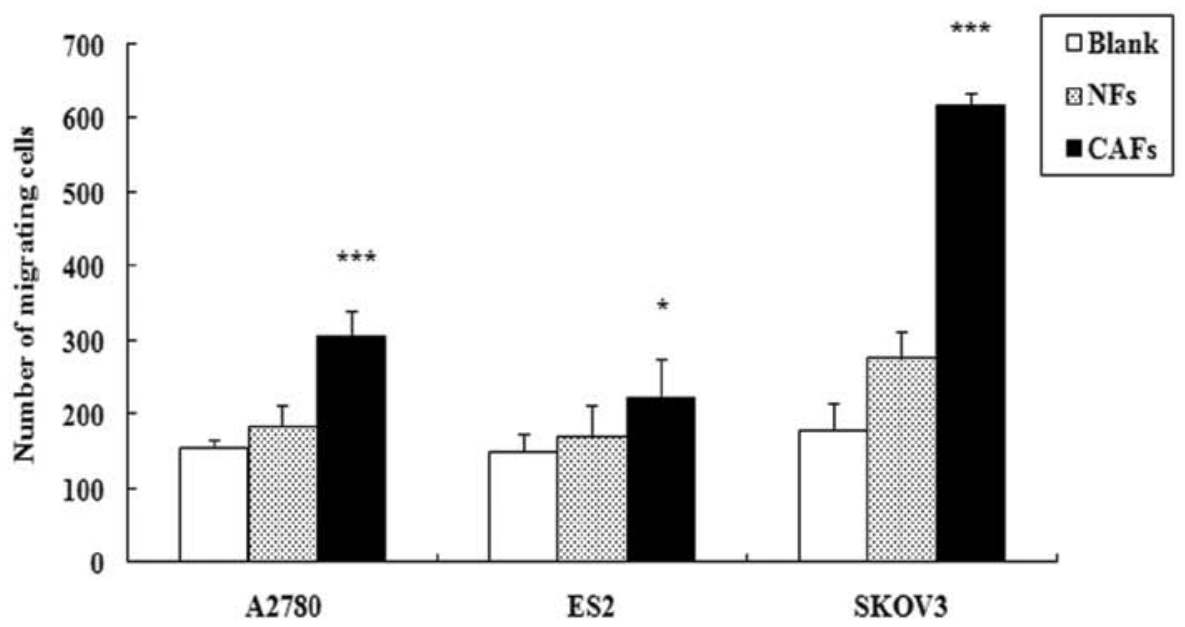

Figure 1. Alterations in the migration ability of ovarian carcinoma cell lines following indirect co-culture with cancer-associated fibroblasts (CAFs) or normal fibroblasts (NFs). (A) Image of cells stained with crystal violet penetrating the membrane, which is based on the high power field (x200) of an inverted fluorescence microscope. The number of ovarian cancer cells indirectly co-cultured with CAFs penetrating the membrane was significantly higher than that of cells cultured alone and cells indirectly co-cultured with NFs. (B) Statistical analysis of the mean number of stained cells penetrating the membrane, which revealed that the number of ovarian cancer cells directly co-cultured with CAFs penetrating the membrane was significantly greater than that of cells cultured alone and cells indirectly co-cultured with NFs; the difference was statistically significant. ${ }^{* * *} \mathrm{P}<0.001$ and ${ }^{*} \mathrm{P}<0.05$ vs. blank group.

the mRNA expression of EZH2 in the ovarian cancer cells (Fig. 2A). Similarly, western blot analysis revealed that the CAFs significantly increased the protein expression of EZH2 in the ovarian cancer cells. Compared with cells cultured alone, the protein expression of EZH2 in the A2780, SKOV3 and ES2 cells co-cultured with CAFs increased by 2.25-, 2.1- and 1.94-fold, respectively $\left(\mathrm{P}_{\mathrm{A} 2780}<0.001, \mathrm{P}_{\mathrm{SKOV} 3}<0.001\right.$ and $\left.\mathrm{P}_{\mathrm{ES} 2}<0.001\right)$; the ovarian NFs did not significantly increase the protein expression of EZH2 in the ovarian cancer cells (Fig. 2B).
$C A F$ s enhance the migration ability of ovarian cancer cells by increasing EZH2 expression. The expression levels of EZH2 in the A2780-shEZH2, A2780-shNC and A2780 cells were assessed by qRT-PCR and western blot analysis. The mRNA expression of EZH2 decreased by approximately $78.2 \%$ in the A2780-shEZH2 cells $(\mathrm{P}<0.001)$ (Fig. 3A), whereas there was no significant difference between the A2780-shNC and A2780 cells (Fig. 3A). The knockdown of EZH2 in the A2780shEZH2 cells was further confirmed by western blot analysis 

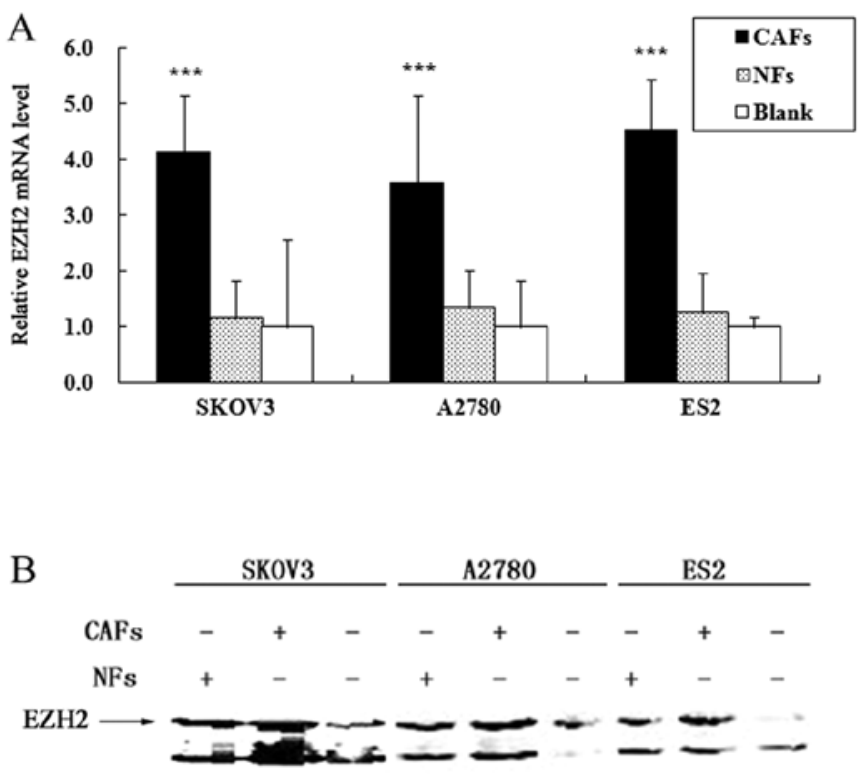

$\beta-\operatorname{actin}$

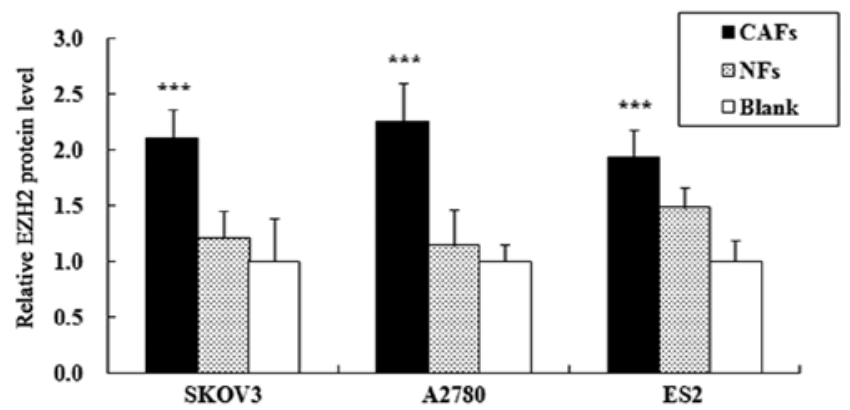

Figure 2. The expression levels of enhancer of zeste homologue 2 (EZH2) in the co-cultured ovarian carcinoma cells were assessed by qRT-PCR and western blot analysis. (A) The relative mRNA level of EZH2 in the co-cultured ovarian cancer cells was assessed by qRT-PCR, and $\beta$-actin was used as a loading control. Cancer-associated fibroblasts (CAFs) significantly increased the relative mRNA level of EZH2 in ovarian cancer cells compared with the normal fibroblasts (NFs) and the cells cultured alone, and the difference was statistically significant. (B) The level of EZH2 protein in the co-cultured ovarian cancer cells was assessed by western blot analysis, and $\beta$-actin was used as a loading control. CAFs significantly increased the level of EZH2 protein in ovarian cancer cells compared with the NFs and the cells cultured alone, and the difference was statistically significant. ${ }^{* * *} \mathrm{P}<0.001$ vs. blank group.

$(\mathrm{P}<0.001)$ (Fig. 3B). Compared with the cells cultured alone, the expression of EZH2 in the A2780-shEZH2 cells co-cultured with CAFs was not significantly increased (P>0.05) (Fig. 4). The number of A2780-shEZH2 cells co-cultured with CAFs penetrating the membrane was 1.26 -fold in comparison to the cells cultured alone, and the number of A2780-shEZH2 cells co-cultured with NFs penetrating the membrane was 1.11-fold in comparison to the cells cultured alone. Transwell assay demonstrated that the CAFs did not enhance the migration ability of the A2780-shEZH2 cells compared with the blank group ( $\mathrm{P}>0.05$ ) (Fig. 5); the CAFs and NFs enhanced
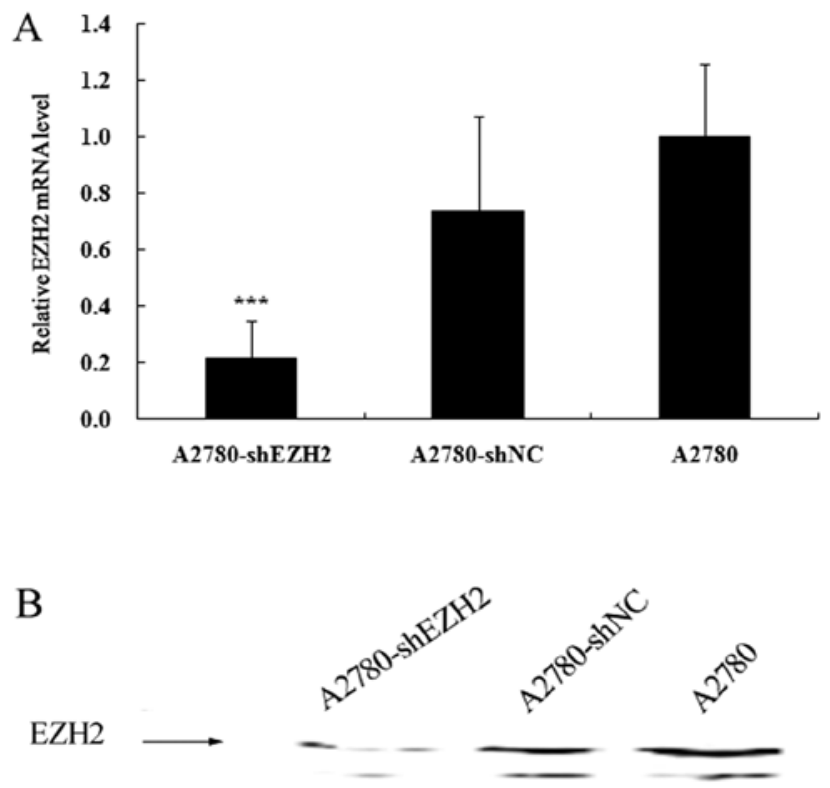

$\beta$-actin

Figure 3. The expression levels of enhancer of zeste homologue 2 (EZH2) in A2780-shEZH2, A2780-shNC and A2780 cells were assessed by qRT-PCR and western blot analysis. (A) The mRNA expression of EZH2 was decreased in the A2780-shEZH2 cells compared with the A2780-shNC cells and the untransfected A2780 cells, as shown by qRT-PCR. (B) The downregulation of EZH2 protein in the A2780-shEZH2 cells compared with the A2780shNC cells and untransfected A2780 cells, as shown by western blot analysis. ${ }^{* * * *} \mathrm{P}<0.001$ vs. A2780-shNC cells and untransfected A2780 cells.
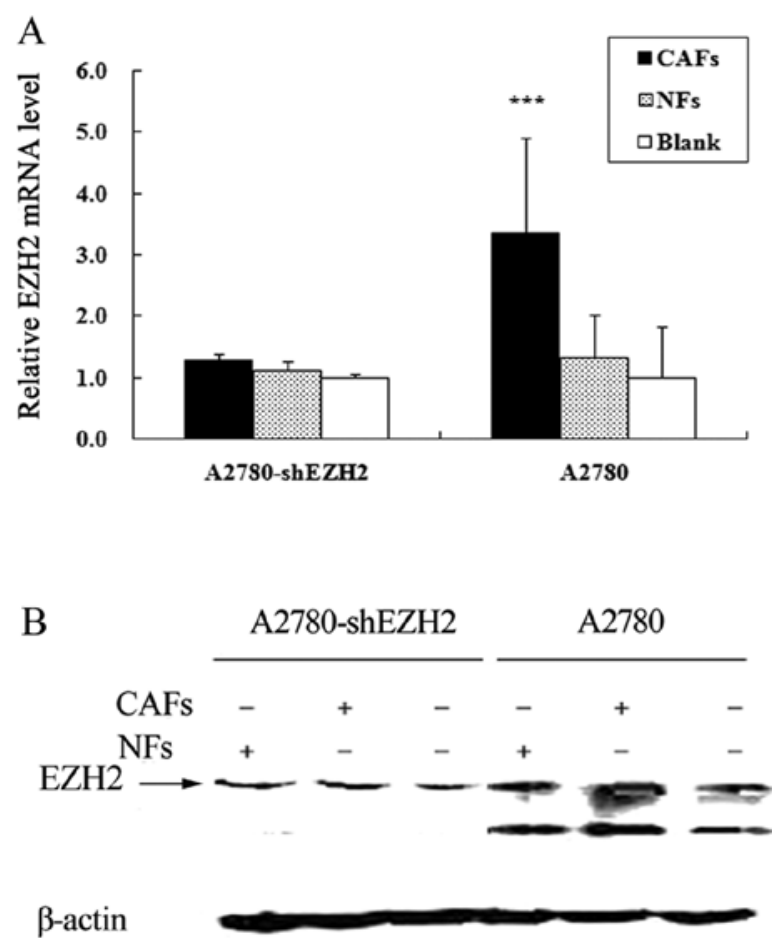

Figure 4. Results of qRT-PCR and western blot analysis for the enhancer of zeste homologue 2 (EZH2) expression in A2780-shEZH2 cells indirectly co-cultured with cancer-associated fibroblasts (CAFs) or normal fibroblasts (NFs). CAFs did not significantly increase the expression of EZH2 in the A2780-shEZH2 cells compared with the NFs and the cells cultured alone $(\mathrm{P}>0.05)$, and CAFs significantly increased the expression of EZH2 in the A2780 cells compared with the NFs and cells cultured alone $(\mathrm{P}<0.001) .{ }^{* * *} \mathrm{P}<0.001$ vs. blank group. 


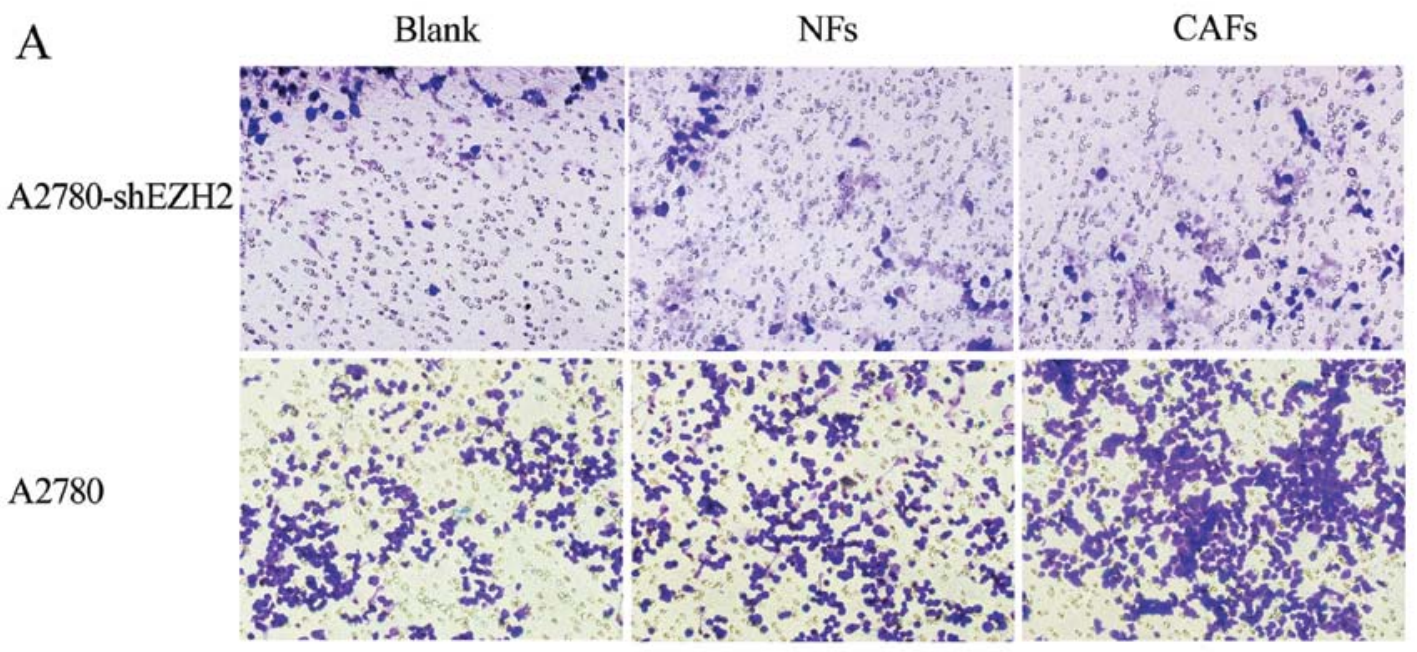

B

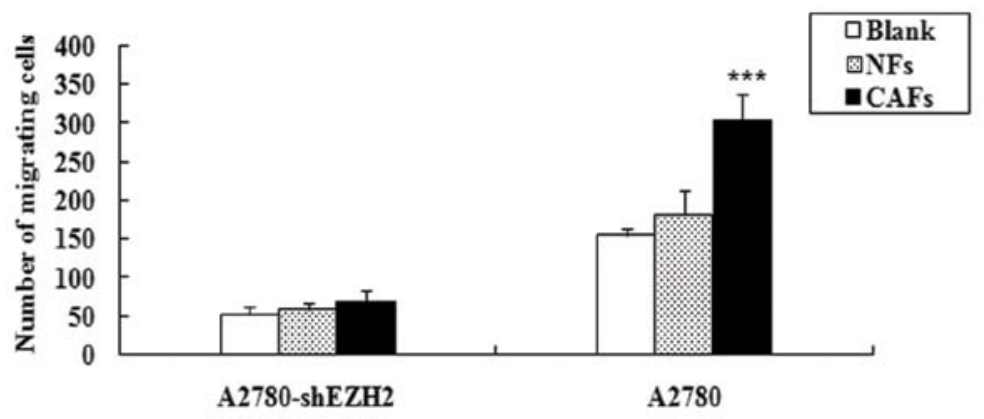

Figure 5. The alterations in the migration ability of A2780-shEZH2 and A2780 cells indirectly co-cultured with cancer-associated fibroblasts (CAFs) or normal fibroblasts (NFs) were detected. (A) The results revealed that the number of A2780-shEZH2 cells indirectly co-cultured with CAFs penetrating the membrane was not significantly higher than that of cells cultured with NFs and the cells cultured alone ( $\mathrm{P}>0.05)$. (B) The number of A2780-shEZH2 cells indirectly cocultured with CAFs penetrating the membrane was not significantly higher than that of cells cultured with NFs and cells cultured alone $(\mathrm{P}>0.05)$. ${ }^{* * * *} \mathrm{P}<0.001 \mathrm{vs}$. blank group. EZH2, enhancer of zeste homologue 2.

the migration ability of the A2780 cells, and the expression of EZH2 in the A2780 cells was much higher than that in the A2780-shEZH2 cells.

\section{Discussion}

The tumor microenvironment is thought to affect malignant transformation and tumor progression. The immune cells in the tumor microenvironment can be quantitatively assessed through epigenetic markers, providing a novel method for the early diagnosis of cancer (17). What is more, cancer epigenetics may be regulated by the tumor microenvironment (such as hypoxia) (18). It has been reported that CAFs promote the growth and invasion of cancer cells; however, the specific mechanisms involved remain unclear (19-21). EZH2 is overexpressed in many types of cancer and plays a crucial role in the tumorigenesis of several types of human cancer (12-14). In ovarian cancer, the overexpression of EZH2 promotes cell proliferation and invasion (22). The aim of this study was to investigate the effects of CAFs on the migration ability of ovarian cancer cells and to determine the involvement of EZH2 in this process, as well as the underlying mechanisms.
In the present study, we found that the migration ability of ovarian cancer cells indirectly co-cultured with CAFs or NFs was significantly enhanced, particularly that of cells indirectly co-cultured with CAFs. Additionally, CAFs significantly increased the mRNA and protein expression of EZH2 in ovarian cancer cells. However, the NFs did not significantly increase the expression of EZH2 in ovarian cancer cells. We therefore considered that CAFs may induce epigenetic alterations in cancer cells. Moreover, CAFs did not enhance the migration ability of the A2780-shEZH2 cells compared with the cells cultured alone.

The exact mechanisms responsible for CAFs enhancing the migration ability of ovarian cancer cells may include two aspects: CAFs induced the upregulation of EZH2 in ovarian cancer cells through paracrine or autocrine mechanisms, subsequently enhancing the migration ability of the ovarian cancer cells; CAFs and EZH2 interacted with each other, which enhanced the migration ability of the ovarian cancer cells.

Our study revealed that ovarian CAFs enhanced the migration ability of the ovarian cancer cells by increasing the expression of EZH2. CAFs enhanced the expression of EZH2 in ovarian cancer cells, which may be due to the function of 
certain factors secreted by CAFs. Several studies have found that certain genes are upreglated in CAFs in contrast to NFs, such as growth factors associated with metastasis [plateletderived growth factor (PDGF)-A, fibroblast growth factor 1 (FGF1), transforming growth factor (TGF)- $\beta$ and interleukin (IL)-6] and factors promoting angiogenesis [vascular endothelial growth factor (VEGF), cyclooxygenase (COX)-2, vimentin and $\alpha$-smooth muscle actin ( $\alpha$-SMA)] $(23,24)$. CAFs may provide the most suitable microenvironment for the growth of ovarian cancer cells. Lu et al confirmed that VEGF induced the upregulation of EZH2 in ovarian cancer cells (25).

In conclusion, our results suggest that CAFs enhance the migration ability of ovarian cancer cells partly by increasing EZH2 expression. We established the link between epigenetic mechanisms and the microenvironment. Further studies are required to identify the mechanisms responsible for CAFs increasing the expression of EZH2 in ovarian cancer cells.

\section{Acknowledgements}

We would like to thank the Department of Obstetrics and Gynecology, Union Hospital, Wuhan, China. This study was supported by the National Natural Science Foundation of China (81072134 and 81272860).

\section{References}

1. Sarojini S, Tamir A, Lim H, et al: Early detection biomarkers for ovarian cancer. J Oncol 2012: 709049, 2012.

2. Bijron JG, Bol GM, Verheijen RH and van Diest PJ: Epigenetic biomarkers in the diagnosis of ovarian cancer. Expert Opin Med Diagn 6: 421-438, 2012

3. Naora $\mathrm{H}$ and Montell DJ: Ovarian cancer metastasis: integrating insights from disparate model organisms. Nat Rev Cancer 5: 355-366, 2005.

4. Chou J and Werb Z: MicroRNAs play a big role in regulating ovarian cancer-associated fibroblasts and the tumor microenvironment. Cancer Discov 2: 1078-1080, 2012.

5. Mitra AK, Zillhardt M, Hua Y, et al: MicroRNAs reprogram normal fibroblasts into cancer-associated fibroblasts in ovarian cancer. Cancer Discov 2: 1100-1108, 2012.

6. Finak G, Bertos N, Pepin F, et al: Stromal gene expression predicts clinical outcome in breast cancer. Nat Med 14: 518-527, 2008.

7. Wintzell M, Hjerpe E, Avall Lundqvist E and Shoshan M: Protein markers of cancer-associated fibroblasts and tumor-initiating cells reveal subpopulations in freshly isolated ovarian cancer ascites. BMC Cancer 12: 359, 2012.
8. Ko SY, Barengo N, Ladanyi A, et al: HOXA9 promotes ovarian cancer growth by stimulating cancer-associated fibroblasts. J Clin Invest 122: 3603-3617, 2012

9. Schauer IG, Sood AK, Mok S and Liu J: Cancer-associated fibroblasts and their putative role in potentiating the initiation and development of epithelial ovarian cancer. Neoplasia 13: 393-405, 2011.

10. Zhang Y, Tang H, Cai J, et al: Ovarian cancer-associated fibroblasts contribute to epithelial ovarian carcinoma metastasis by promoting angiogenesis, lymphangiogenesis and tumor cell invasion. Cancer Lett 303: 47-55, 2011.

11. Cai J, Tang H, Xu L, et al: Fibroblasts in omentum activated by tumor cells promote ovarian cancer growth, adhesion and invasiveness. Carcinogenesis 33: 20-29, 2012.

12. Cao R, Wang L, Wang H, et al: Role of histone H3 lysine 27 methylation in Polycomb-group silencing. Science 298: 1039-1043, 2002.

13. Kirmizis A, Bartley SM, Kuzmichev A, et al: Silencing of human polycomb target genes is associated with methylation of histone H3 Lys 27. Genes Dev 18: 1592-1605, 2004.

14. Simon JA and Lange CA: Roles of the EZH2 histone methyltransferase in cancer epigenetics. Mutat Res 647: 21-29, 2008.

15. Hu S, Yu L, Li Z, et al: Overexpression of EZH2 contributes to acquired cisplatin resistance in ovarian cancer cells in vitro and in vivo. Cancer Biol Ther 10: 788-795, 2010.

16. Livak KJ and Schmittgen TD: Analysis of relative gene expression data using real-time quantitative PCR and the 2(-Delta Delta C(T)) Method. Methods 25: 402-408, 2001.

17. Sehouli J, Loddenkemper C, Cornu T, et al: Epigenetic quantification of tumor-infiltrating T-lymphocytes. Epigenetics 6: 236-246, 2011.

18. Shahrzad S, Bertrand K, Minhas K and Coomber BL: Induction of DNA hypomethylation by tumor hypoxia. Epigenetics 2: 119-125, 2007.

19. Kalluri R and Zeisberg M: Fibroblasts in cancer. Nat Rev Cancer 6: 392-401, 2006.

20. Shimoda M, Mellody KT and Orimo A: Carcinoma-associated fibroblasts are a rate-limiting determinant for tumour progression. Semin Cell Dev Biol 21: 19-25, 2010.

21. Xing F, Saidou J and Watabe K: Cancer associated fibroblasts (CAFs) in tumor microenvironment. Front Biosci (Landmark Ed) 15: 166-179, 2010.

22. Li H, Cai Q, Godwin AK and Zhang R: Enhancer of zeste homolog 2 promotes the proliferation and invasion of epithelial ovarian cancer cells. Mol Cancer Res 8: 1610-1618, 2010.

23. Matsumoto K and Nakamura T: Hepatocyte growth factor and the Met system as a mediator of tumor-stromal interactions. Int J Cancer 119: 477-483, 2006.

24. Orimo A, Gupta PB, Sgroi DC, et al: Stromal fibroblasts present in invasive human breast carcinomas promote tumor growth and angiogenesis through elevated SDF-1/CXCL12 secretion. Cell 121: 335-348, 2005.

25. Lu C, Han HD, Mangala LS, et al: Regulation of tumor angiogenesis by EZH2. Cancer Cell 18: 185-197, 2010. 